\title{
The effect of TCAP on acquisition of discrimination learning in the rat
}

DENVER DANIELS ${ }^{1}$

UNIVERSITY OF BRISTOL

The effect of TCAP, a compound which increases CNS, RNA and protein availability, on rat $S$ s acquisition of a visual discrimination $\mathrm{T}$ maze situation was studied. Acute administration of $20 \mathrm{mg} / \mathrm{kg}$ TCAP by i.p. injection resulted in a significant improvement in acquisition in "dull" and "average" Ss but no such improvement occurred in the "bright" Ss.

Previous studies have indicated that TCAP (1.1.3.tricyano-2-amino-1-propene ${ }^{2}$ ) stimulates an increase in nerve cell RNA and protein within the Central Nervous System (CNS) (Egyhazi \& Hyden, 1961) and facilitates the acquisition of simple adaptive responses (Chamberlain et al, 1963) and of passive avoidance responses (Essman, 1965). Although such evidence provides some support for Hyden's hypothesis (1959) that RNA and protein are involved in the acquisition process, at least in simple learning situations, Chamberlain et al (1963) using a Hebb-Williams maze situation, did not find that an injection of TCAP facilitated complex learning. More recently Brush et al (1966) showed that TCAP did not facilitate either avoidance learning or acquisition in a 6-unit water maze. The present study was designed to investigate the effect of TCAP on "dull" "average" and "bright" rats in a visual $T$ maze situation.

\section{Subjects}

Ss were 37 male albino rats of Wistar ancestry whose free-feeding weights ranged from 250 to $300 \mathrm{gm}$. Procedure

All Ss were reduced to $85 \%$ of their free-feeding body weight prior to introduction to the experimental situation, in which lighting, handling and orientation of the maze were all held constant. Ss were pretrained in a black runway. They were tested on a first visual discrimination $\mathrm{T}$ maze and on the basis of their score divided into "dull" (error score $>17 ; \mathrm{N}=11$ ), "average" (error score 6-16; $\mathrm{N}=13$ ) and "bright" (error score< $5 ; N=13$ ). They were then tested in a second visual discrimination $T$ maze to assess the effects of TCAP. The two mazes were "calibrated" to be of equal difficulty as follows. Three Ss were randomly selected from each group and introduced to the second maze where they returned error scores which were not significantly different from those they had achieved in the first maze. These nine Ss took no further part in the experiment.

Each group of Ss-"dull," "average" and "bright"was randomly but equally divided into control and experimental subgroups. Ss in the experimental subgroups received $20 \mathrm{mg} / \mathrm{kg}$ TCAP at $37^{\circ} \mathrm{C}$ by intraperi- toneal injection $45 \mathrm{~min}$. prior to introduction to the second maze. Control subgroups similarly received an equal volume of $0.85 \%$ physiological saline. After each $S$ had achieved the criterion, which was 18 correct responses in 20 trials, the maze was washed for $2 \mathrm{~min}$. in hot running water to minimize olfactory cues. A measure of the time spent by each $S$ in the maze was recorded in minutes (Maze Time), together with error scores (the number of times a $S$ entered the wrong arm of the maze) and the number of trials necessary to achieve criterion.

It was predicted that error scores and the number of trials needed to achieve criterion would be significantly lower in the experimental (TCAP) subgroups than in control subgroups.

Results

Ss in the "dull" TCAP subgroup made significantly fewer errors in the second maze $(p=0.014$, one-tailed) and required fewer trials to achieve criterion $(p=0.014$, one-tailed) than their control subgroup. Ss in the "average" TCAP subgroup also made fewer errors $(p=0.048$, one-tailed) and needed fewer trials $(p=0.028$, onetailed) than their control subgroups to achieve criterion. In the "bright" group no significant difference was found between TCAP and control subgroups in either error scores or number of trials. No significant difference was demonstrable in Maze Times between any subgroups.

Figures 1, 2, and 3 show the mean number of errors in "dull" "average" and "bright" groups in successive blocks of 10 trials to criterion.

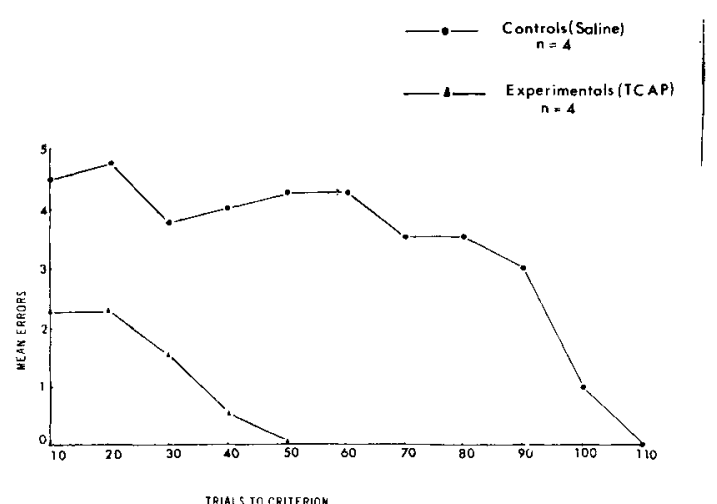

Fig. 1. "Dull" Group. Data from second visual discrimination $\mathbf{T}$ maze showing mean error scores in successive blocks of ten trials up to criterion. 


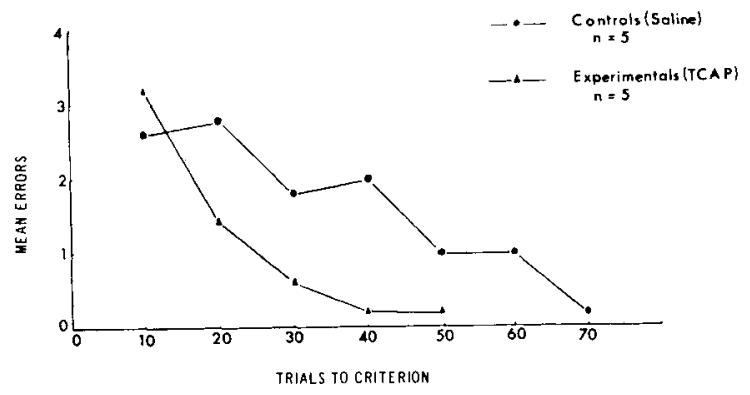

Fig. 2. "Average" Group. Data from second visual discrimination $T$ maze showing mean error scores in successive blocks of ten trials up to criterion.

\section{Discussion}

The results show that acquisition in "dull" and "average" rats can be significantly improved by acute administration of TCAP which chemically stimulates an increase in CNS nerve cell RNA and protein availability.

The graphs in Figs. 1, 2, and 3 show a gradual decrease in the effect achieved by TCAP between groups, and this together with the failure to demonstrate a

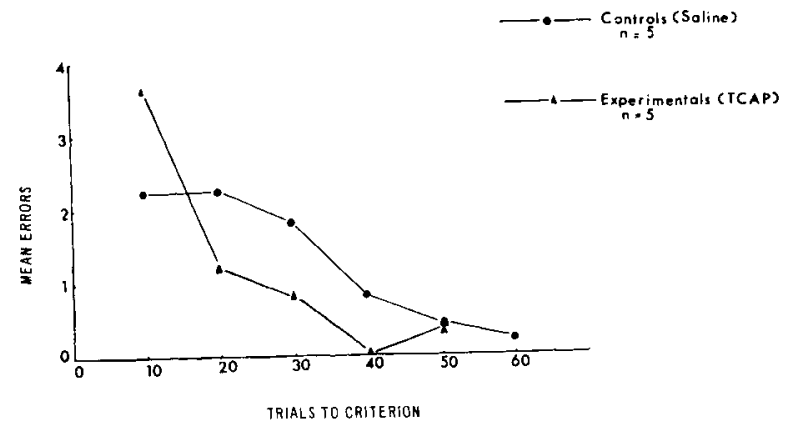

Fig. 3. "Bright" Group. Data from second visual discrimination $T$ maze showing mean error scores in successive blocks of ten trials up to criterion. significant improvement in the "bright" Ss suggests that CNS nerve cell RNA and protein availability cannot be raised above a certain limit and the "bright" Ss were already functioning at this limit.

The discrepancy between the present findings and those of Chamberlain et al (1963) and Brush et al (1966) who were unable to demonstrate facilitation of acquisition in complex learning situations may be understood when it is pointed out that Ss in the two studies quoted were not divided into "dull," "average" and "bright" groups and differential drug effects in Ss would become submerged in the final evaluation of the results. Secondly both previous studies used a different drug dosage, 15 instead of $20 \mathrm{mg} / \mathrm{kg}$ and Brush etal (1966) also used chronic rather than acute adminstration of TCAP.

Although the involvement of RNA and protein in the acquisition process is further evidenced by these results, their specific roles remain to be demonstrated.

\section{References}

Brush, F. R., Davenport, J. W., \& Polidora, v. J. TCAP: Negative results in avoidance and water maze learning and retention. Psychon. Sci., 1966, 4, 183-184.

Chamberlain, T. J., Rothschild, G. H., \& Gerard, R. W. Drugs affecting RNA and learning. Proc. Nat. Acad. Sci., 1963, 49, 918-924.

Essman, W. B. Facilitation of memory consolidation by chemically induced acceleration of RNA synthesis. In Upjohn Research Laboratory Summary, March 22,1965 . P. 98.

Egyhazi, E., \& Hyden, H. Experimentally induced changes in the base composition of the ribonucleic acids of isolated nerve cells and their Oligodendroglial cells. J. Biophys. Biochem. Cytol., 1961, 10, 403-410.

Hyden, H. 4th International Congress of Biochemistry - Proceedings. $1959,3,64-89$.

Siegel, S. Nonparametric statistics. New York: McGraw-Hill and Kogakusha, 1956.

\section{Notes}

1. I wish to thank Dr. I. W. Pleydell-Pearce for providing laboratory facilities and Dr. S. M. Anstis and Dr. D. R. Haslam for their helpful comments.

2. 1.1.3, tricyano-2-amino-1-propene was provided by the Upjohn Co. Kalamazoo, Michigan. 